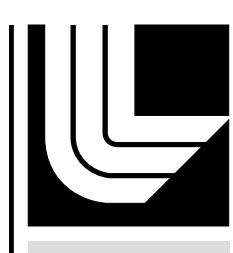

LAWRENCE LIVERMORE NATIONAL LABORATORY

\title{
Monitoring Spent or \\ Reprocessed Nuclear Fuel Using Fast Neutrons
}

G. F. Chapline, L. F. Nakae, N. Snyderman, J. M. Verbeke, R. Wurtz

May 31, 2011

ICENES

San Francisco, CA, United States

May 15, 2011 through May 19, 2011 
This document was prepared as an account of work sponsored by an agency of the United States government. Neither the United States government nor Lawrence Livermore National Security, LLC, nor any of their employees makes any warranty, expressed or implied, or assumes any legal liability or responsibility for the accuracy, completeness, or usefulness of any information, apparatus, product, or process disclosed, or represents that its use would not infringe privately owned rights. Reference herein to any specific commercial product, process, or service by trade name, trademark, manufacturer, or otherwise does not necessarily constitute or imply its endorsement, recommendation, or favoring by the United States government or Lawrence Livermore National Security, LLC. The views and opinions of authors expressed herein do not necessarily state or reflect those of the United States government or Lawrence Livermore National Security, LLC, and shall not be used for advertising or product endorsement purposes. 


\title{
Monitoring Spent or Reprocessed Nuclear Fuel Using Fast Neutrons
}

\author{
G. F. Chapline, L. F. Nakae, N. Snyderman, J. M. Verbeke, R. Wurtz \\ Lawrence Livermore National Laboratory: P.O. Box 808,Livermore, CA 94551 \\ chapline1@llnl.gov
}

Over the past few years a number of experiments have been carried out at LLNL with a scintillator array that has the ability to count individual MeV neutrons and $\gamma$ rays with nanosecond timing. It has been demonstrated that this array can be used to measure the statistical properties of the neutrons emitted in single fission chains. The multiple time scales over which these fission neutrons are correlated allow one to deduce quite a lot regarding the nature of the fissile assembly. In this paper we will describe how neutron correlations measured with a liquid scintillator array can be used to assay the amounts of fissile elements in reprocessed and spent nuclear fuels.

\section{Historical Background}

The basic theory of neutron fluctuations and time correlations in near critical assemblies of fissionable materials was developed during WWII by R. Feynman and E. Schrödinger. The most important prediction is that a modest amount of multiplication leads to long fission chains and highly non-Poisson neutron count distributions. Feynman and de Hoffmann (1944). developed a formula for relating the excess neutron intensity fluctuation relative to Poisson distribution

$$
\frac{<c^{2}-\bar{c}^{2}>}{\bar{c}}-1 \equiv 2 Y_{2 F}
$$

in a homogeneous sub-critical assembly to the neutron multiplication $M$ and neutron lifetime $1 / \alpha$ inside the assembly. In particular, they showed that

$$
\begin{gathered}
Y_{2 F}=\varepsilon\left(M-\frac{M-1}{\bar{v}}\right)\left(\frac{v_{2 s}}{\bar{v}_{s}}+(M-1) \frac{v_{2 i}}{\bar{v}_{i}}\right) \\
*\left\lfloor 1-\frac{1-e^{-\alpha t}}{\alpha t}\right\rfloor,
\end{gathered}
$$

where $\varepsilon$ is the detector efficiency, and $v$ and $v_{2}$ are the average numbers of neutrons and neutron pairs in a single fission ${ }^{1}$. The indices $s$ and $i$ refer to spontaneous and induced fissions. This formula agreed with measurements of $Y_{2 F}$ for the "Water Boiler Reactor" carried out at Los Alamos in 1944 by E. Segre.
If the detector efficiency $\varepsilon$ is unknown, then Eq. (1) must be supplemented with additional information in order to determine the multiplication. One possibility is to use the 3-neutron Feynman correlation function $Y_{3 \mathrm{~F}}$ (Ref 2). In the decades following WW II determining the multiplication of sub-critical assemblies using ${ }^{3} \mathrm{He}$ neutron detectors to measure the 2- and 3-neutron correlation functions $Y_{2 \mathrm{~F}}$ and $Y_{3 \mathrm{~F}}$ became a cottage industry at LANL. However, because ${ }^{3} \mathrm{He}$ neutron detectors can only see thermal neutrons it is difficult to distinguish individual fission chains or distinguish the effects of multiple time scales in a non-homogeneous assembly. These deficiencies have been overcome with development of neutron detectors that can directly detect fission neutrons with nanosecond timing.

\section{Fast Neutron Counting}

In 2008, W. Stöffl et al built a liquid scintillator array that uses liquid xylene and has the ability to count individual $\mathrm{MeV}$ neutrons and $\gamma$-rays with nanosecond timing resolution ${ }^{3}$. A number of experiments have been carried out with this array and its descendants that demonstrate its usefulness for directly seeing the multiple neutrons emitted in a single fission chain.

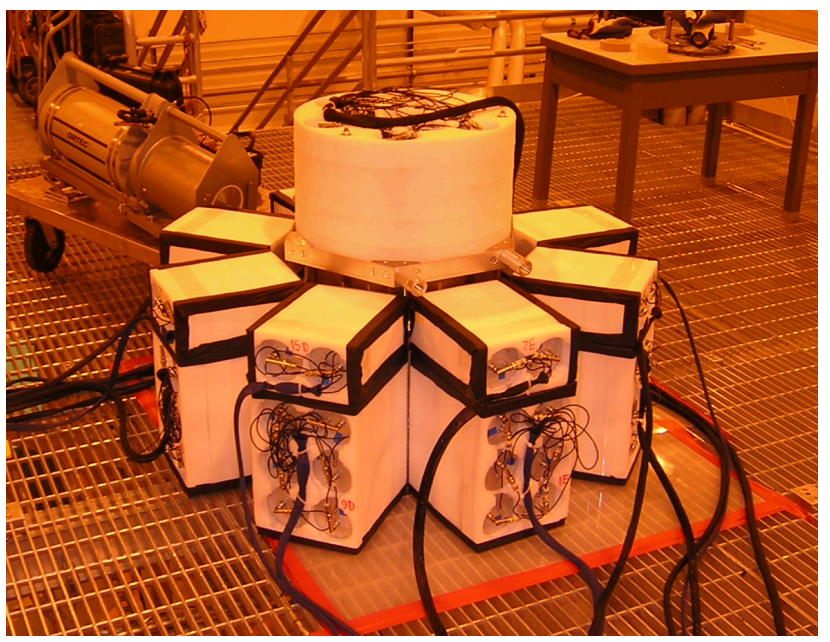

Fig. 1. Liquid scintillator array with fast electronic readout used in LLNL experiments 
One type of problem where fast neutron counting has turned out to be particularly useful is the analysis of nonhomogeneous fissile material assemblies, for example a fissile material assembly surrounded by a layer of moderating material. The neutron multiplication $M$ of a fissile assembly surrounded by an external moderator will be larger than that of the "bare" fissile assembly because neutrons that are thermalized in the external moderator can be reflected back into the fissile assembly, producing additional fission chains. If the assembly contains isotopes with a large thermal neutron cross-section, e.g. ${ }^{235} \mathrm{U}$ or ${ }^{239} \mathrm{Pu}$, the increase in the multiplication can be significant. The Feynman 2-neutron correlation function including these "restart" fissions is given by:

$$
Y_{2 F}=\left(\frac{M_{0}}{M}\right)^{2} Y_{2 F}\left(\alpha_{1} t\right)+\left(1-\frac{M_{0}^{2}}{M^{2}}\right) Y_{2 F}\left(\alpha_{2} t\right) \text {, }
$$

where the $Y_{2 \mathrm{~F}}(\alpha \mathrm{t})$ functions are similar in form to the homogeneous Feynman-de Hoffmann formula (1). This formula works particularly well in cases where the time scales $1 / \alpha_{1}$ and $1 / \alpha_{2}$ are very different. This happens to be the case, for example, when a homogeneous fissile material is surrounded by a distinct thin moderating shell.

\section{Assaying the Pu in MOX Fuel}

One example of a situation where one might hope that the formula (2) is useful is a nuclear fuel assembly surrounded by a shell of moderating material such as polyethylene. We have obtained evidence that this expectation is correct by carrying out Monte Carlo calculations using MCNP for simulated fuel assemblies surrounded by a cylindrical array of liquid scintillators.

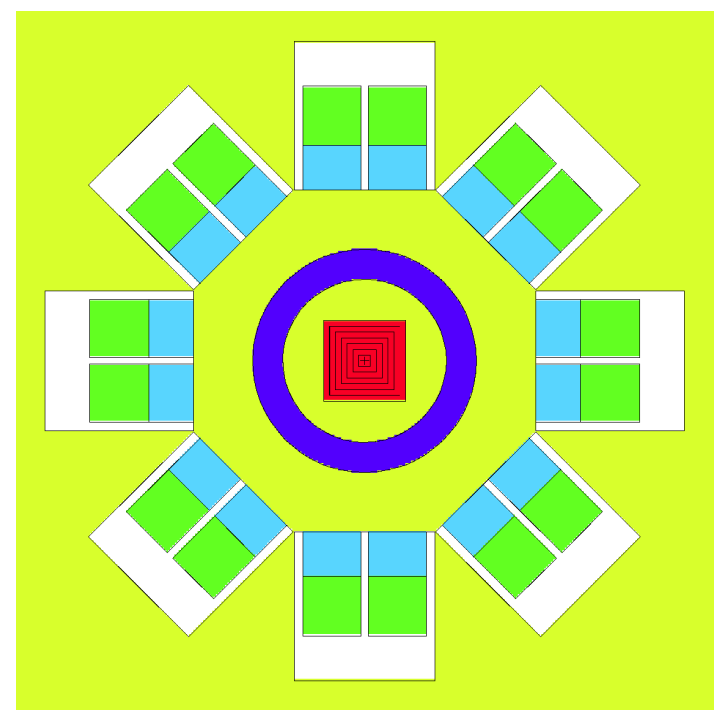

Fig. 2. Geometry used in Monte Carlo calculations.

The geometry used in the calculations is shown in Fig. 2. We assumed that the nuclear fuel (indicated in red) has a square cross-section of side $14 \mathrm{~cm}$ and is $1 \mathrm{~m}$ long. The polyethylene moderator (indicated in blue) was assumed to be a cylindrical shell $5 \mathrm{~cm}$ thick over the length of the assembly. The cylindrical detector array was $1.4 \mathrm{~m}$ long and contained $19210-\mathrm{cm}$ diameter, $7.5-\mathrm{cm}$ deep xylene cells. The actinide compositions used in our simulations are shown in Table 1. The observing time was typically 100 seconds.

We obtained the 2-neutron correlation function $Y_{2 \mathrm{~F}}$ by analyzing the statistics of the fast neutrons recorded in the cylindrical stack of liquid scintillators. Our strategy is to use the simulated 2-neutron and 3-neutron correlations for time intervals less than a microsecond to determine the rate of generation of spontaneous neutrons and the "bare" multiplication $M$.

In Fig 3 we show the results of a Monte Carlo calculation of the Feynman correlation function $Y_{2 \mathrm{~F}}$ for $125 \mathrm{~kg}$ of $\mathrm{MOX}$ fuel with no $\mathrm{CH}_{2}$ layer.

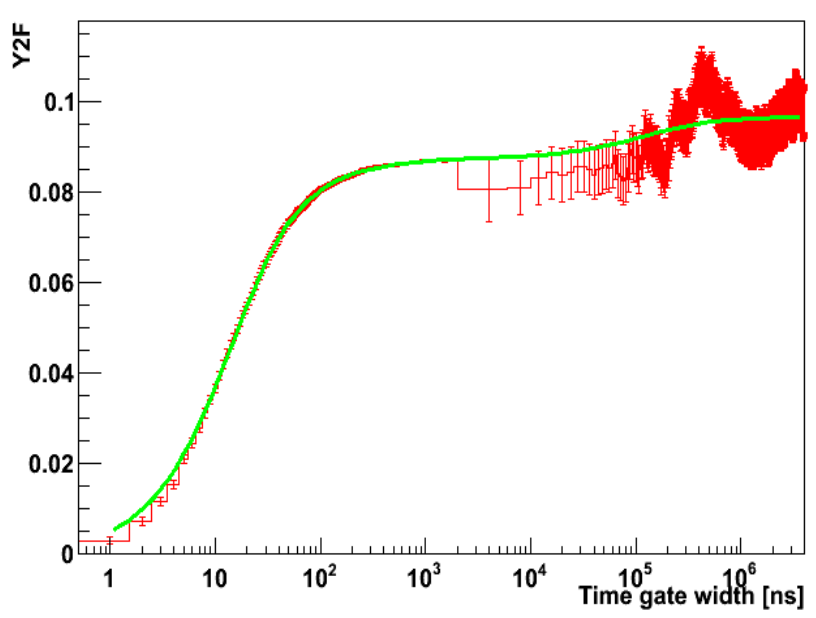

Fig. 3. Monte Carlo $Y_{2 \mathrm{~F}}$ for $125 \mathrm{~kg}$ MOX fuel with no surrounding $\mathrm{CH}_{2}$ moderator.

Our simulated MOX fuel contained no ${ }^{242} \mathrm{Cm}$ or ${ }^{244} \mathrm{Cm}$, so the predominant source of spontaneous fission neutrons is ${ }^{240} \mathrm{Pu}$. The values for the detector efficiency and "bare" multiplication we obtained from our Monte Carlo calculations of $Y_{2 \mathrm{~F}}$ (shown in Fig. 3) and $Y_{3 \mathrm{~F}}$ for the 125 $\mathrm{kg}$ of MOX fuel with no external $\mathrm{CH}_{2}$ moderator are $\varepsilon=$ $7.5 \%$ and $M_{o}=1.17$. The source strength of spontaneous fission neutrons we infer from the observed count rate, together with the inferred values of $M_{\mathrm{o}}$ and $\varepsilon$, is completely consistent with the spontaneous fission rate expected from the $0.6 \mathrm{~kg}$ of ${ }^{240} \mathrm{Pu}$ in $125 \mathrm{~kg}$ of MOX fuel.

Given $M_{\mathrm{o}}$ the amount of ${ }^{235} \mathrm{U}+{ }^{239} \mathrm{Pu}+{ }^{241} \mathrm{Pu}$ in the MOX fuel can be inferred from the behavior of the 2neutron correlation function $Y_{2 \mathrm{~F}}$ for time intervals on the order of milliseconds when the fuel assembly is surrounded by an external $\mathrm{CH}_{2}$ moderator. Fig 4 shows the 2-neutron correlations we obtained from our Monte 
Carlo calculations when the MOX fuel is surrounded by a 5-cm thick $\mathrm{CH}_{2}$ layer. Assuming $M_{o}=1.17$, the detector efficiency and total multiplication $M$ obtained from the Monte Carlo calculation of $Y_{2 \mathrm{~F}}$ for the MOX fuel with a $5 \mathrm{~cm} \mathrm{CH}_{2}$ layer are $\varepsilon=3.5 \%$ and $M=1.54$. It is expected the bare multiplications are the same in the no moderating layer and moderated cases. Again, the source strength inferred from the observed count rate is consistent with the spontaneous fission rate expected from the $0.6 \mathrm{~kg}$ of ${ }^{240} \mathrm{Pu}$ in $125 \mathrm{~kg}$ of MOX fuel.

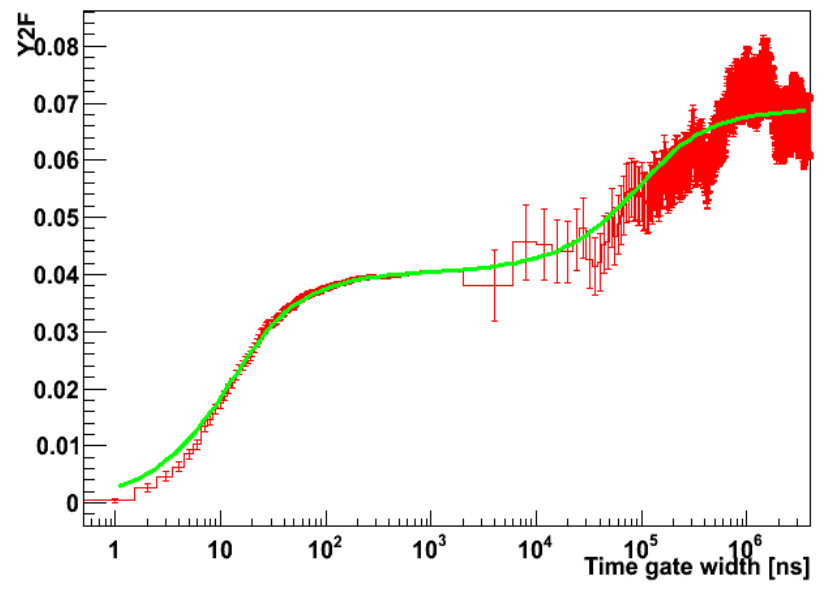

Fig. 4 Monte Carlo $Y_{2 \mathrm{~F}}$ for $125 \mathrm{~kg}$ MOX fuel surrounded by a $5 \mathrm{~cm} \mathrm{CH}_{2}$ layer.

The amount of ${ }^{239} \mathrm{Pu}+{ }^{241} \mathrm{Pu}$ in the MOX nuclear fuel can be inferred by comparing the asymptotic value for $Y_{2 \mathrm{~F}}$ for moderation times (milliseconds) with the plateau value for $Y_{2 \mathrm{~F}}$ for times on the order of a microsecond. As is evident from Eq. (2), the ratio of these plateau values is $\left(M / M_{0}\right)^{2}$. The excess of $\mathrm{M}$ over $\mathrm{M}_{0}$ is due to the contribution of fissions in the ${ }^{239} \mathrm{Pu},{ }^{241} \mathrm{Pu}$, and ${ }^{235} \mathrm{U}$ induced by thermalized neutrons. The value $M=1.54$ we obtained is consistent with the amounts of these isotopes assumed in the simulation.

From a safeguards perspective, one way of masking the diversion of $\mathrm{Pu}$ would be to replace the $\mathrm{Pu}$ with a small amount of ${ }^{252} \mathrm{Cf}$. If the $4 \mathrm{~kg}$ of $\mathrm{Pu}$ in our $125 \mathrm{~kg}$ of MOX fuel is replaced by $25 \mu \mathrm{g}$ of ${ }^{252} \mathrm{Cf}$, the spontaneous neutron rate will be approximately the same. Figs. 5 and 6 show the results of Monte Carlo calculations for the 2neutron Feynman correlation functions with $25 \mu \mathrm{g}$ of ${ }^{252} \mathrm{Cf}$ replacing the $\mathrm{Pu}$ and with and without a $5 \mathrm{~cm}$ layer of $\mathrm{CH}_{2}$. It can be seen that when there is no $\mathrm{CH}_{2}$ moderator, the $Y_{2 \mathrm{~F}}$ curve is flatter for millisecond times. If we assume that the "bare" multiplication remains the same when the $\mathrm{Pu}$ is replaced by $\mathrm{Cf}$, then the multiplication inferred from the ratio of the time $>$ msec plateau to the short time plateau is 1.3 versus 1.54 in the case when $\mathrm{Pu}$ is present. The removal of the $\mathrm{Pu}$ is reflected in the decrease of the neutron multiplication.

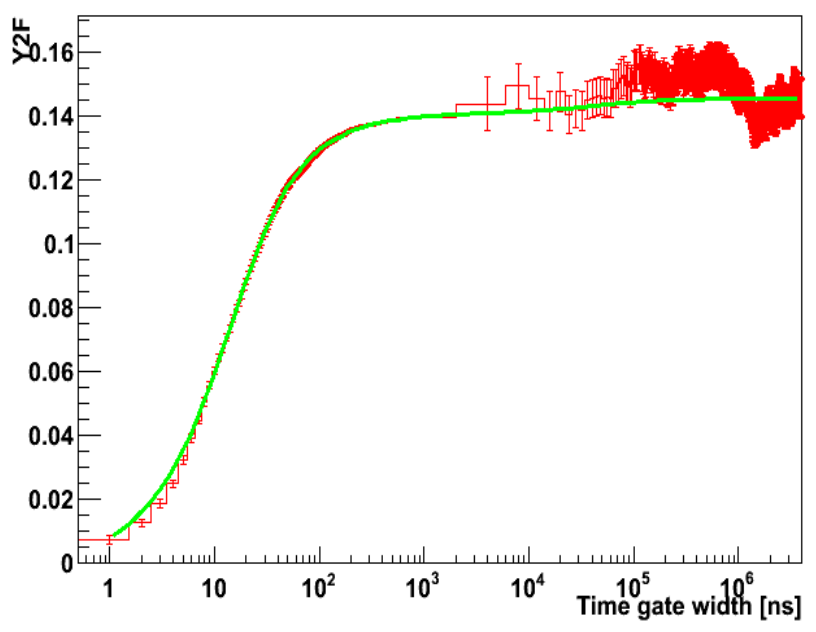

Fig. 5 Monte Carlo $Y_{2 \mathrm{~F}}$ for $125 \mathrm{~kg}$ MOX fuel with ${ }^{252} \mathrm{Cf}$ replacing the $\mathrm{Pu}$ and no $\mathrm{CH}_{2}$ layer.

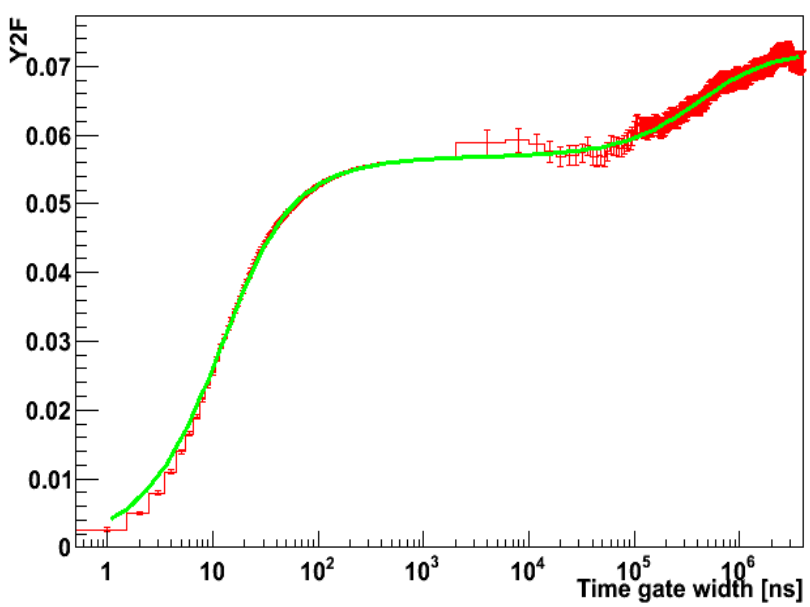

Fig. 6. Monte Carlo $Y_{2 \mathrm{~F}}$ for $125 \mathrm{~kg}$ MOX fuel with ${ }^{252} \mathrm{Cf}$ replacing the $\mathrm{Pu}$, but surrounded by a $5 \mathrm{~cm} \mathrm{CH}_{2}$ layer.

TABLE I. Actinide masses used in simulations $(\mathrm{kg})$

\begin{tabular}{|l|l|l|}
\hline Isotopes & MOX fuel & Spent fuel \\
\hline${ }^{235} \mathrm{U}$ & 0.7 & 20 \\
\hline${ }^{238} \mathrm{U}$ & 105 & 125 \\
\hline${ }^{239} \mathrm{Pu}$ & 3.4 & 1.4 \\
\hline${ }^{240} \mathrm{Pu}$ & 0.6 & 0.5 \\
\hline${ }^{241} \mathrm{Pu}$ & 0.2 & 0.2 \\
\hline${ }^{241} \mathrm{Am}$ & none & 0.04 \\
\hline${ }^{242} \mathrm{Cm}$ & none & 0.002 \\
\hline${ }^{244} \mathrm{Cm}$ & none & 0.004 \\
\hline
\end{tabular}




\section{Assaying the Pu and ${ }^{235} \mathrm{U}$ in Spent Reactor Fuel}

The case of spent fuel differs from MOX fuel in that the predominant source of spontaneous fission neutrons is ${ }^{242} \mathrm{Cm}$ and ${ }^{244} \mathrm{Cm}$ rather than ${ }^{240} \mathrm{Pu}$. In addition, a thick high $\mathrm{Z}$ shield must be placed between the liquid scintillator array and the fuel in order to shield the liquid scintillator array from the enormous $\gamma$-ray background due to the fission products in the spent fuel. Our hope for using neutron correlation techniques to analyze the ${ }^{235} \mathrm{U}$ and $\mathrm{Pu}$ content in spent fuel lies in the idea that a high $\mathrm{Z}$ shield that is thick enough to reduce the $\gamma$-ray flux to acceptable levels, might still be thin enough to measure fission neutron correlations.

In Figs. 7-8, we show the results of Monte Carlo calculations of $Y_{2 F}$ and $Y_{3 F}$ for a section of a simulated assembly of fuel rods not surrounded by any $\mathrm{CH}_{2}$ with an assumed actinide composition typical for spent reactor fuel with $33000 \mathrm{MWd} /$ ton burn-up,. We assumed that the liquid scintillators could only view a $1 \mathrm{~m}$ section of the fuel rod assembly, and that they were shielded with $10 \mathrm{~cm}$ of $\mathrm{Pb}$. This thickness of $\mathrm{Pb}$ corresponds to about 1 neutron mean free path for fission neutrons.

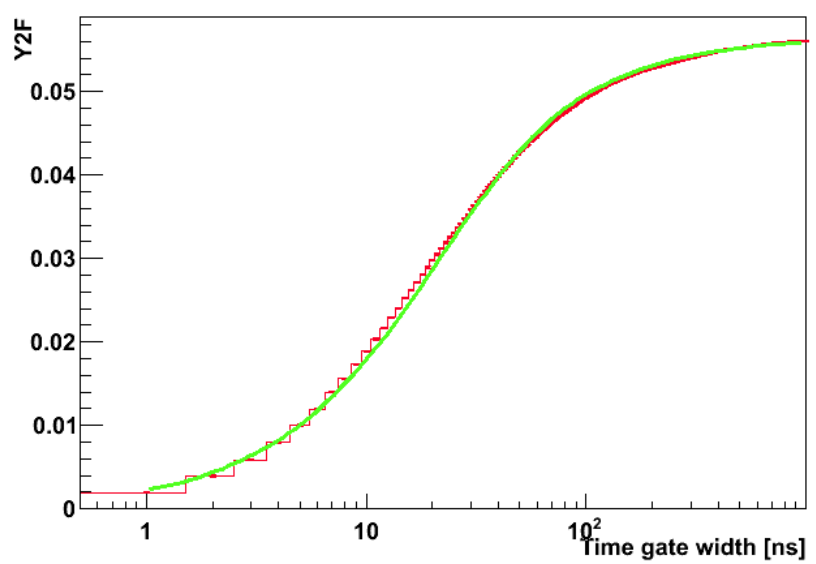

Fig. 7. Monte Carlo $Y_{2 \mathrm{~F}}$ for spent fuel shielded with $10 \mathrm{~cm}$ $\mathrm{Pb}$ and no $\mathrm{CH}_{2}$ layer.

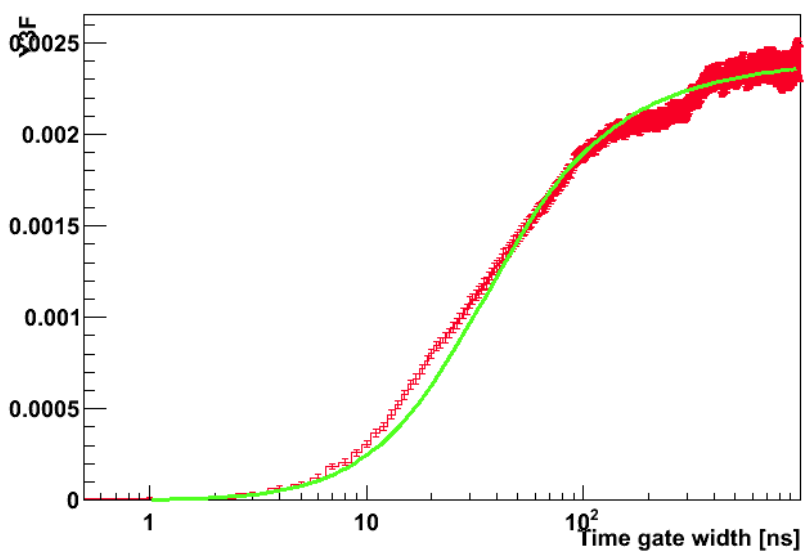

Fig. 8. Monte Carlo $Y_{3 \mathrm{~F}}$ for spent fuel shielded with $10 \mathrm{~cm}$ $\mathrm{Pb}$, but no $\mathrm{CH}_{2}$ layer.
As was the case with MOX fuel, we can use $Y_{2 F}$ and $Y_{3 F}$ to obtain the spontaneous neutron rate and the bare multiplication $M_{0}$ of the spent fuel assembly. The detector efficiency $\varepsilon$ was determined to be approximately $2 \%$ and the bare multiplication $M_{0}=1.1$. The observed count rate in this case was $3.5 .10^{6} \mathrm{sec}^{-1}$, which is consistent with the assumed amounts of ${ }^{242} \mathrm{Cm}$ and ${ }^{244} \mathrm{Cm}$.

In Fig. 9, we show the results of Monte Carlo calculations of $\mathrm{Y}_{2 \mathrm{~F}}$ when the spent fuel is shielded with 5 $\mathrm{cm}$ of $\mathrm{CH}_{2}$ and $10 \mathrm{~cm}$ of $\mathrm{Pb}$. The total multiplication $M$ is 1.6. As was the case with MOX fuel, the excess of $M$ over $M_{0}$ is due to the fissions induced in ${ }^{239} \mathrm{Pu}+{ }^{241} \mathrm{Pu}+{ }^{235} \mathrm{U}$ by thermal neutrons.

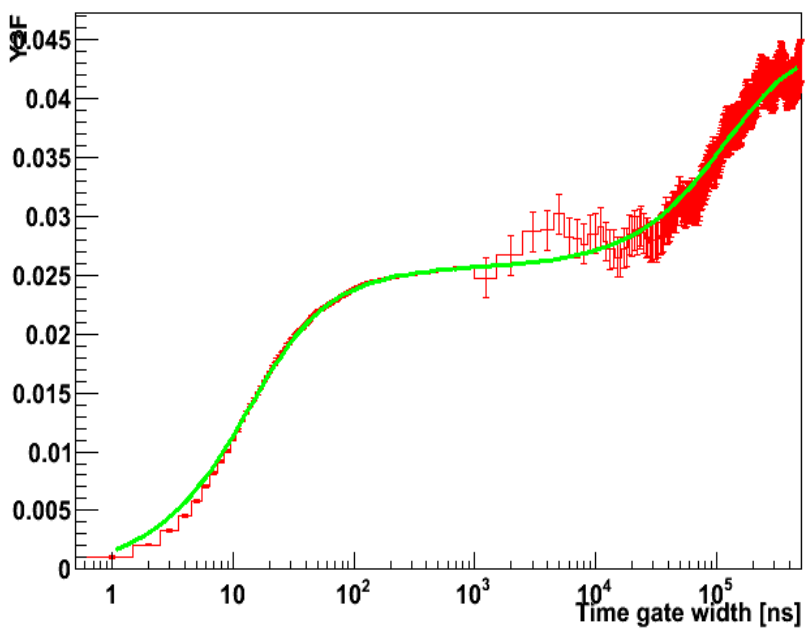

Fig. 9. Monte Carlo $Y_{2 \mathrm{~F}}$ for spent fuel shielded with $5 \mathrm{~cm}$ $\mathrm{CH}_{2}$ and $10 \mathrm{~cm} \mathrm{~Pb}$.

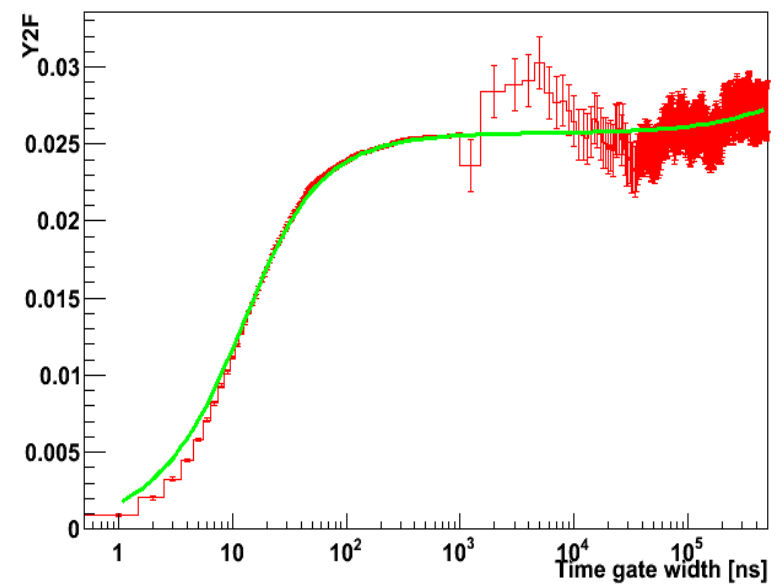

Fig. 10. Monte Carlo $Y_{2 \mathrm{~F}}$ for same fuel configuration, but with the $\mathrm{Pu}$ and ${ }^{235} \mathrm{U}$ removed.

In order to get some idea whether it is possible to use our methods to determine the amounts of $\mathrm{Pu}$ and ${ }^{235} \mathrm{U}$ remaining in spent fuel rods after they have been removed 
from service, we have repeated the spent fuel Monte Carlo calculation with the $\mathrm{Pu}$ and ${ }^{235} \mathrm{U}$ removed. The results for $Y_{2 F}$ are shown in Fig 10. The multiplication in this case is 1.3 versus 1.6 with no diversion of $\mathrm{Pu}$ and ${ }^{235} \mathrm{U}$. The difference between the average count rate with and without $\mathrm{Pu}$ and ${ }^{235} \mathrm{U}$ is a factor 1.5 . The ratio of multiplications tells us that $\approx 30 \%$ of the increase in count rate is due to induced fissions in the ${ }^{239} \mathrm{Pu}+{ }^{241} \mathrm{Pu}+{ }^{235} \mathrm{U}$. In principle, by using Monte Carlo calculations to map the multiplication $M$ to the combined mass of these isotopes, one could determine this mass by measuring $Y_{2 F}$.

In Fig. 11, we show a calculation of $Y_{2 F}$ for the shielded spent fuel with only the $\mathrm{Pu}$ removed. The second shoulder in $Y_{2 F}$, is relatively lower than the one in Fig. 9, which indicates that the total multiplication $M$ is reduced. We estimate the total multiplication $M$ in this case to be 1.5 versus 1.6 with no diversion of $\mathrm{Pu}$. Although the results do appear to be sensitive to amount of $\mathrm{Pu}$, it is not clear at this point whether we can determine the individual masses of the ${ }^{239} \mathrm{Pu},{ }^{241} \mathrm{Pu}$, or ${ }^{235} \mathrm{U}$ isotopes. This will require further study.

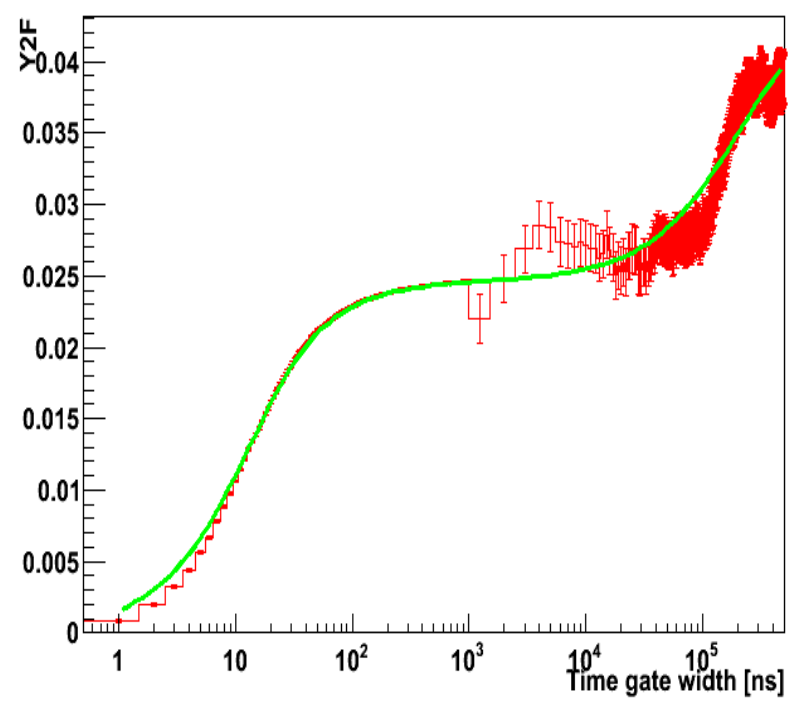

Fig. 11. Monte Carlo $Y_{2 \mathrm{~F}}$ for spent fuel shielded with $5 \mathrm{~cm}$ $\mathrm{CH}_{2}$ and $10 \mathrm{~cm} \mathrm{~Pb}$, but containig no $\mathrm{Pu}$.

\section{CONCLUSIONS}

Our Monte Carlo simulation results suggest that fast neutron counting techniques ought to be very useful for assaying the amounts of fissile materials in spent and reprocessed nuclear fuel. In particular the short time behavior of $Y_{2 \mathrm{~F}}$ and $Y_{3 \mathrm{~F}}$ can be used to assay the amounts of ${ }^{240} \mathrm{Pu}$ in the reprocessed fuel and $\mathrm{Cm}$ in spent fuel. If the reprocessed fuel is surrounded with a few inches of moderator, then the long time behavior of $Y_{2 \mathrm{~F}}$ can be used to quite accurately determine the absolute amount of ${ }^{239} \mathrm{Pu}$ $+{ }^{241} \mathrm{Pu}$ in the reprocessed fuel. In the case of spent fuel the neutron counting statistics are somewhat marginal due to the need to use a high $\mathrm{Z}$ shield thick enough to block the enormous $\gamma$-ray flux. However, with a $\mathrm{Pb}$ shield about 1 neutron mean free thick, the statistics are good enough to at least estimate the total amount of ${ }^{239} \mathrm{Pu}+{ }^{241} \mathrm{Pu}+{ }^{235} \mathrm{U}$ in the spent fuel. We are still looking into whether we can identify the amounts of $\mathrm{Pu}$ isotopes.

\section{ACKNOWLEDGMENTS}

This work performed under the auspices of the U.S. Department of Energy by Lawrence Livermore National Laboratory under Contract DE-AC52-07NA27344.

\section{REFERENCES}

1. R. P. FEYNMAN, F. DE HOFFMANN, R. SERBER, "Dispersion of the Neutron Emission in U235 Fission," J. of Nuclear Energy 3, 64, (1956).

2. W. HAGE, and D. M. CIFARELLI, "Models for a Three-Parameter Analysis of Neutron Signal Correlation Measurements for Fissile Material Assay," Nucl. Instr. and Meth. A251, 550 (1986).

3. R. WURTZ et al, "Verification with $\gamma$-ray and Fast Neutron Detection," Proc. of INMM, Baltimore, MD, July 2010, 51, INMM (2010). 